\title{
Early reperfusion therapy in acute ischemic stroke after recent myocardial infarction
}

Otimizando os benefícios da terapia de reperfusão precoce no AVC agudo após infarto agudo do miocárdio

Gustavo Wruck Kuster1,2, Antonio Claudio Baruzzi', Evelyn de Paula Pacheco 1,2, Renan Barros Domingues 1,2, Marco Pieruccetti², Luciano Marcus Tirotti Giacon², Jose Carlos Teixeira Garcia', Valter Furlan', Ayrton Roberto Massaro ${ }^{3}$

A 50-year-old woman with recent myocardial infarction (MI), presented right hemiparesis with aphasia (National Institutes of Health Stroke Scale: 18), 24 hours after the coronary angiogram that revealed a large apical thrombus (Figure 1A). Computed tomography demonstrated left middle cerebral artery (MCA) "dot" sign (Figure 1B). Angiography showed occlusion in the MCA second segment (Figure 1C) with recanalization after mechanical thrombectomy (Figure 1D). She presented a complete recovery (Rankin:0) and after 9 days she was discharged with oral anticoagulation. Recent MI within previous 3 months is a contraindication to IV thrombolysis (myocardial rupture) ${ }^{1,2}$. Mechanical thrombolysis seems to be a safe and effective alternative in this situation ${ }^{3,4,5}$.

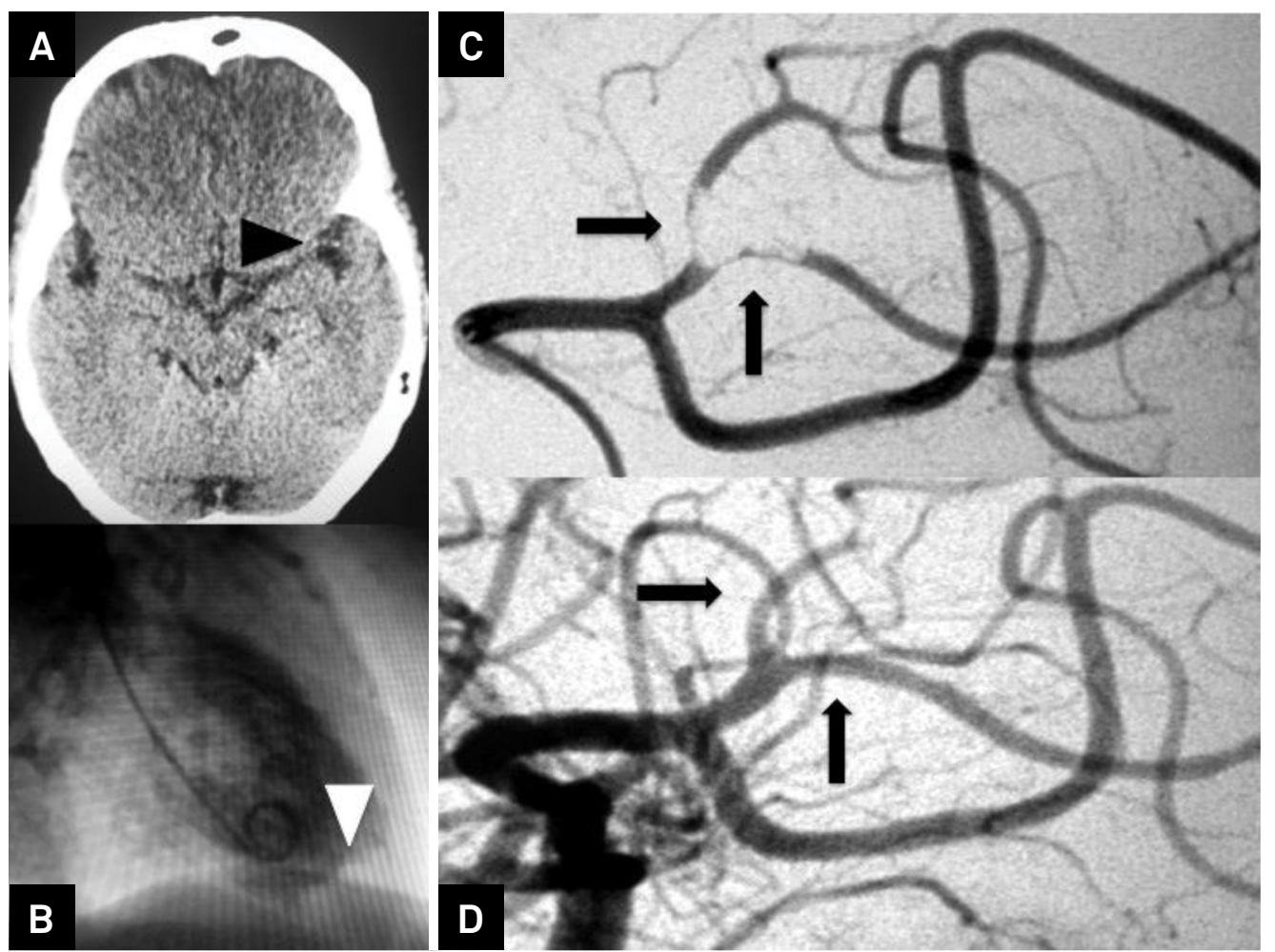

Figure 1. (A) Computed tomography demonstrated a left middle cerebral artery "dot" sign (arrowhead); (B) Coronary angiogram revealed an apical myocardial disfunction with a large thrombus; (C) Cerebral angiogram demonstrated an occlusion;

(D) Recanalization after an endovascular procedure using a mechanical device (Penumbra system).

\footnotetext{
${ }^{1}$ Hospital Total Cor, São Paulo SP, Brasil;

${ }^{2}$ Hospital Paulistano, São Paulo SP, Brasil;

${ }^{3}$ Hospital Sírio Libanês, São Paulo SP, Brasil.

Correspondence: Gustavo W Kuster; Rua Morgado de Mateus, 259 / apto 11;04015-050 São Paulo SP, Brasil; E-mail: gwkuster@hotmail.com Conflict of interes: There is no conflict of interest to declare.

Received 29 August 2015; Accepted 16 May 2016
} 


\section{References}

1. Martins SCO, Freitas GR, Pontes-Neto OM, et al. Guidelines for acute ischemic stroke treatment - Part II: Stroke treatment. Arq Neuropsiquiatr. 2012;70(11):885 -93. doi:10.1590/S0004-282X2012001100012

2. Jauch EC, Saver JL, Adams Jr HP, Bruno A, Connors JJ, Demaerschalk BM et al. Guidelines for the early management of patients with an acute ischemic stroke: a guideline for Healthcare professionals from the American Heart Association/ American Stroke Association. Stroke. 2013;44(3):870-947. doi:10.1161/STR.0b013e318284056a

3. Hussain SI, Zaidat OO, Fitzsimmons BF. The Penumbra system for mechanical thrombectomy in endovascular acute ischemic stroke therapy. Neurology. 2012;79(13 Suppl 1):135-41. doi:10.1212/WNL.0b013e31826958a8

4. Widimsky P, Coram R, Abou-Chebi A. Reperfusion therapy of acute ischaemic stroke and acute myocardial infarction: similarities and differences. Eur Heart J. 2014;35(3):147-55. doi:10.1093/eurheartj/eht409

5. Singer OC, Haring HP, Trenkler J, Nolte CH, Bohner G, Neumann-Haefelin T et al. Periprocedural aspects in mechanical recanalization for acute stroke: data from the ENDOSTROKE registry. Neuroradiology. 2013;55(9):1143-51. doi:10.1007/s00234-013-1219-9 